\title{
Stability and folding properties of a model $\beta$-sheet protein, Escherichia coli CspA
}

\author{
KATHERINE L. REID, HECTOR M. RODRIGUEZ, BRIAN J. HILLIER, AND LYDIA M. GREGORET \\ Department of Chemistry \& Biochemistry, University of California, Santa Cruz, California 95064
}

\begin{abstract}
Although $\beta$-sheets represent a sizable fraction of the secondary structure found in proteins, the forces guiding the formation of $\beta$-sheets are still not well understood. Here we examine the folding of a small, all $\beta$-sheet protein, the $E$. coli major cold shock protein CspA, using both equilibrium and kinetic methods. The equilibrium denaturation of CspA is reversible and displays a single transition between folded and unfolded states. The kinetic traces of the unfolding and refolding of CspA studied by stopped-flow fluorescence spectroscopy are monoexponential and thus also consistent with a two-state model. In the absence of denaturant, CspA refolds very fast with a time constant of $5 \mathrm{~ms}$. The unfolding of CspA is also rapid, and at urea concentrations above the denaturation midpoint, the rate of unfolding is largely independent of urea concentration. This suggests that the transition state ensemble more closely resembles the native state in terms of solvent accessibility than the denatured state. Based on the model of a compact transition state and on an unusual structural feature of CspA, a solvent-exposed cluster of aromatic side chains, we propose a novel folding mechanism for CspA. We have also investigated the possible complications that may arise from attaching polyhistidine affinity tags to the carboxy and amino termini of CspA.
\end{abstract}

Keywords: cold shock; fast protein folding; polyhistidine tags; protein stability; stopped-flow

The challenge to understanding $\beta$-sheet structure is to decipher how residues, which may be distant from one another along the polypeptide chain, locate one another specifically and in a reasonable amount of time. To help elucidate the mechanism of sheet formation, we have characterized the folding and stability of the all $\beta$-sheet protein, CspA, the major cold shock protein of $E$. coli. CspA is an ideal model for $\beta$-sheet folding studies not only because, as shown here, it is well-behaved biophysically, but also because the protein's structure represents a general single-stranded nucleic acid-binding motif. Having a firm grasp of the structurefunction relationship in this protein should aid in future protein design endeavors that could lead to the development of novel therapeutics and other molecular tools.

Progress in understanding $\beta$-structure has, in part, been hampered by the difficulty in developing peptide models for sheets. Initial attempts to make isolated $\beta$-hairpins resulted in peptide aggregation because of the competition between identical intramolecular and inter-molecular interactions, and successful design of hairpins has only recently been reported (Blanco et al., 1994; Searle et al., 1995; Nesloney \& Kelly, 1996; Ramirez-Alvarado et al., 1996).

The folding of several small $\beta$-sheet-containing proteins have been studied by kinetic methods. Although in general it appears

Reprint requests to: Lydia M. Gregoret, Department of Chemistry \& Biochemistry, University of California, Santa Cruz, California 95064; e-mail: gregoret@chemistry.ucsc.edu. that smaller proteins are more likely to fold rapidly without detectable intermediates, the spectrum of folding behaviors for this group of proteins is broad. For example, the immunoglobulinbinding domain of streptococcal protein $\mathrm{G}$ ( 56 amino acids) folds rapidly -with (time constant $(\tau)=5 \mathrm{~ms}$ ) (Alexander et al., 1992), as does the Bacillus subtilis cold shock protein CspB (67 amino acids; $\tau=1 \mathrm{~ms}$ ) (Schindler et al., 1995). The SH3 domain of spectrin (62 amino acids) also folds without detectable intermedjates, though more slowly ( $\tau=350 \mathrm{~ms}$ ) (Viguera et al., 1994). Conversely, ubiquitin ( 76 amino acids) folds very rapidly (in under $3 \mathrm{~ms}$ ), but through an intermediate (Khorasanizadeh et al., 1996). The related " $\beta$ clam" proteins (131 amino acids), cellular retinoic acid binding protein, and intestinal fatty acid binding protein (IFABP) acquire native structure on the second time scale via folding intermediates (Ropson et al., 1990; Clark et al., 1996). Beta conformation is detected early in interleukin $1 \beta$ (153 amino acids), but stable inter-strand hydrogen bonds do not appear until later (Varley et al., 1993). For the majority of molecules (Kiefhaber, 1995), the predominantly helical domain of hen egg white lysozyme (129 amino acids) folds within $200 \mathrm{~ms}$, while the mostly $\beta$-sheet $\beta$-domain takes more than one second to fold (Dobson et al., 1994). Most curiously, the folding of the mostly $\beta$-sheet protein, $\beta$-lactoglobulin ( 162 amino acids), proceeds via an $\alpha$-helical burst-phase intermediate (Hamada et al., 1996) In summary, it does not appear that $\beta$-sheet proteins generally require a folding intermediate or fold more slowly than $\alpha$-helical proteins as initially postulated (Rudolph et al., 1990; Ropson \& Frieden, 1992; Varley 


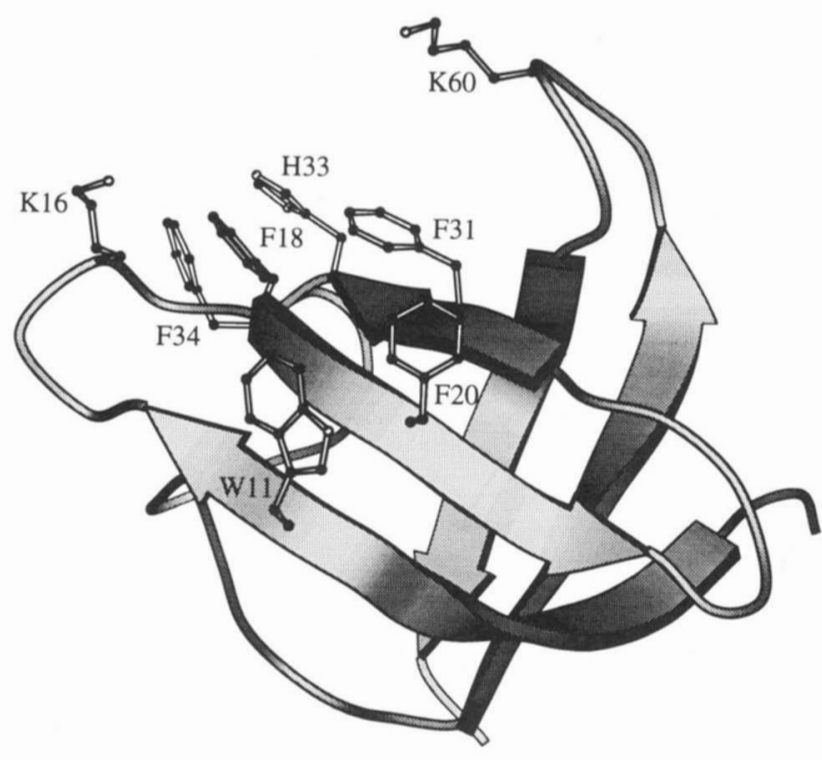

Fig. 1. Ribbon drawing of the cold shock protein CspA from E. coli. Side chains comprising the nucleic acid binding site are shown. This site includes an unusual solvent-exposed aromatic cluster. This representation is from MOLSCRIPT (Kraulis, 1991) based on the coordinates of Schindelin et al. (Schindelin et al., 1994).

et al., 1993; Liu et al., 1994). Rapid acquisition of $\beta$-structure is achievable and, therefore, the question of how $\beta$-sheet proteins fold remains open and all the more enticing. Furthermore, determining how $\beta$-sheets assemble could shed light on the protein folding problem in general.

Here we report on both the equilibrium and kinetic folding of CspA, a small all- $\beta$ protein. This protein is expressed at high levels when bacteria are chilled to $\angle 15^{\circ} \mathrm{C}$ (Goldstein et al., 1990) and is proposed to function as an RNA chaperone (Jiang et al., 1997). CspA is an ideal simple model system for studying protein folding for many reasons. The three-dimensional structure of $\mathrm{CspA}$ (Fig. 1) has been solved by both X-ray crystallography (Schindelin et al., 1994) and nuclear magnetic resonance spectroscopy (Newkirk et al., 1994). CspA contains an unusual cluster of solventexposed aromatic residues that are postulated to participate in nucleic acid binding (Newkirk et al., 1994). Among these residues is a single tryptophan ( $\operatorname{Trp} 11$ ), which can serve as a fluorescent probe for monitoring the folding spectroscopically. Furthermore, CspA has no disulfide bonds, cis peptide bonds, co-factors, or posttranslational modifications that may complicate folding. It is also soluble in the folded and the unfolded state and monomeric. By contrast, B. subtilis $\mathrm{CspB}$, whose folding has been studied in detail (Schindler et al., 1995; Schindler \& Schmid, 1996) dimerizes under certain conditions (Schindelin et al., 1993; Makhatadze \& Maraheil, 1994). Therefore, CspA is potentially a simpler model for investigating folding than $\mathrm{CspB}$.

We have examined the stability of CspA to urea and $\mathrm{GdnHCl}$ denaturation at $25^{\circ} \mathrm{C}$, as well as at $10^{\circ} \mathrm{C}$, the temperature at which CspA functions in vivo. We have also monitored its stability to thermal denaturation by circular dichroism (CD) and fluorescence spectroscopies. The folding kinetics of CspA were examined using stopped-flow fluorescence. Because of the common use of polyhistidine tags for affinity purification of proteins, we have compared wild-type CspA to two variants: CspA, with six histidine residues at the carboxy terminus (CspA-H6), and CspA, with six histidines at the amino terminus followed by a Factor Xa protease cleavage site (H6-Xa-CspA).

Although it is often expected that homologous proteins fold by similar pathways and on similar time scales (Hooke et al., 1994; Kragelund et al., 1996), it cannot be assumed. Case in point: a single-point mutation can dramatically alter the folding or refolding rates of a protein (Matouschek et al., 1995; Milla et al., 1995). Comparison of our data to a recent transition state theorybased analysis of the folding of B. subtilis CspB (Schindler \& Schmid, 1996) indicates that these proteins, which are $58 \%$ identical, $d o$ behave similarly. Although the folding of CspB is faster, both proteins fold via a transition state that closely resembles the native state. We propose that a nucleation site comprised of conserved, solvent-exposed aromatic amino acids stabilizes the coldshock proteins in native-like transition states, enabling the $\beta$-strands to align productively and for the proteins to proceed quickly to their native states. Alignment of aromatic residues may guide the folding of other small $\beta$-sheet proteins as well.

\section{Results}

\section{Equilibrium denaturation followed by fluorescence}

Although the single tryptophan ( Trp 11) in CspA is partially exposed to solvent on the surface of the protein (Fig. 1) (Newkirk et al., 1994; Schindelin et al., 1994), its intrinsic fluorescence emission decreases approximately $50 \%$ from the folded to the unfolded state. The fluorescence emission spectra for the histidinetagged CspA variants are identical (data not shown). The emission maximum of Trp 11 shifts slightly from 349 to $353 \mathrm{~nm}$ upon unfolding as expected for a partially exposed tryptophan, and confirming a previous report (Chatterjee et al., 1993).

Figure $2 \mathrm{~A}$ shows the changes in fluorescence intensity at $349 \mathrm{~nm}$ of the three $\mathrm{CspA}$ variants upon titration with urea at $25^{\circ} \mathrm{C}$. In each case, a single transition between the initial and final states was observed. The three variants exhibited a similar dependence of free energy of unfolding on urea concentration $\left(m_{e q} \approx 0.7\right.$; Table 1$)$. Linear extrapolation of the data results in the free energy of unfolding in the absence of urea $\left(\Delta G_{\mathrm{H}_{2} \mathrm{O}}\right)$ as described by Equation 1. In Table 1, the values for $\Delta G_{\mathrm{H}_{2} \mathrm{O}}, m_{e q}$, and [urea $]_{1 / 2}\left(C_{m}\right)$ are reported for the urea unfolding of the $\mathrm{CspA}$ variants followed by fluorescence at 10 and $25^{\circ} \mathrm{C}$. The unfolding free energy of all three variants at both temperatures is close to $3.0 \mathrm{kcal} / \mathrm{mol}$.

We also determined the change in fluorescence intensity at $349 \mathrm{~nm}$ of the CspA variants upon titration with $\mathrm{GdnHCl}$ at $25^{\circ} \mathrm{C}$ and $10^{\circ} \mathrm{C}$. Again, a single transition was observed between the initial and final states for the variants at both temperatures. The values for $\Delta G_{\mathrm{H}_{2} \mathrm{O}}, m_{e q}$, and $[\mathrm{GdnHCl}]_{1 / 2}$ are listed in Table 2. All three variants had similar $\mathrm{GdnHCl}$ unfolding curves at $25^{\circ} \mathrm{C}$ (Fig. 2B).

\section{Equilibrium denaturation followed by circular dichroism}

The CspA variants exhibited similar far UV-CD spectra at high and low temperatures. The spectrum of wild type CspA is shown in Figure 3. CspA exhibits an unusual far UV-CD spectrum for a predominantly $\beta$-sheet protein. The positive ellipticity in the far-UV region is attributed to the aromatic chromophores positioned on the surface of $\beta$-strands 1, 2, and 3 (Chatterjee et al., 1993) (Fig. 1). 
Table 1. Midpoint of denaturation, $m_{e q}$, and $\Delta G_{\mathrm{H}_{2} \mathrm{O}}$ values for urea denaturation followed by fluorescence at 10 and $25^{\circ} \mathrm{C}$

\begin{tabular}{|c|c|c|c|c|c|c|}
\hline \multirow{2}{*}{$\begin{array}{c}\text { Temperature } \\
\left({ }^{\circ} \mathrm{C}\right)\end{array}$} & \multicolumn{2}{|c|}{ [urea $_{1 / 2}(\mathrm{M})$} & \multicolumn{2}{|c|}{$\Delta G_{\mathrm{H}_{2} \mathrm{O}}(\mathrm{kcal} / \mathrm{mol})$} & \multicolumn{2}{|c|}{$m_{e q}(\mathrm{kcal} / \mathrm{mol} * \mathbf{M})$} \\
\hline & 10 & 25 & 10 & 25 & 10 & 25 \\
\hline Wild-type CspA & $4.9 \pm 0.1$ & $4.2 \pm 0.1$ & $2.9 \pm 0.1$ & $3.0 \pm 0.1$ & $0.59 \pm 0.05$ & $0.71 \pm 0.04$ \\
\hline H6-Xa-CspA & $5.0 \pm 0.2$ & $4.2 \pm 0.1$ & $3.3 \pm 0.2$ & $2.9 \pm 0.1$ & $0.66 \pm 0.04$ & $0.69 \pm 0.05$ \\
\hline CspA-H6 & $4.5 \pm 0.2$ & $4.2 \pm 0.1$ & $2.8 \pm 0.2$ & $3.2 \pm 0.2$ & $0.64 \pm 0.05$ & $0.78 \pm 0.06$ \\
\hline
\end{tabular}
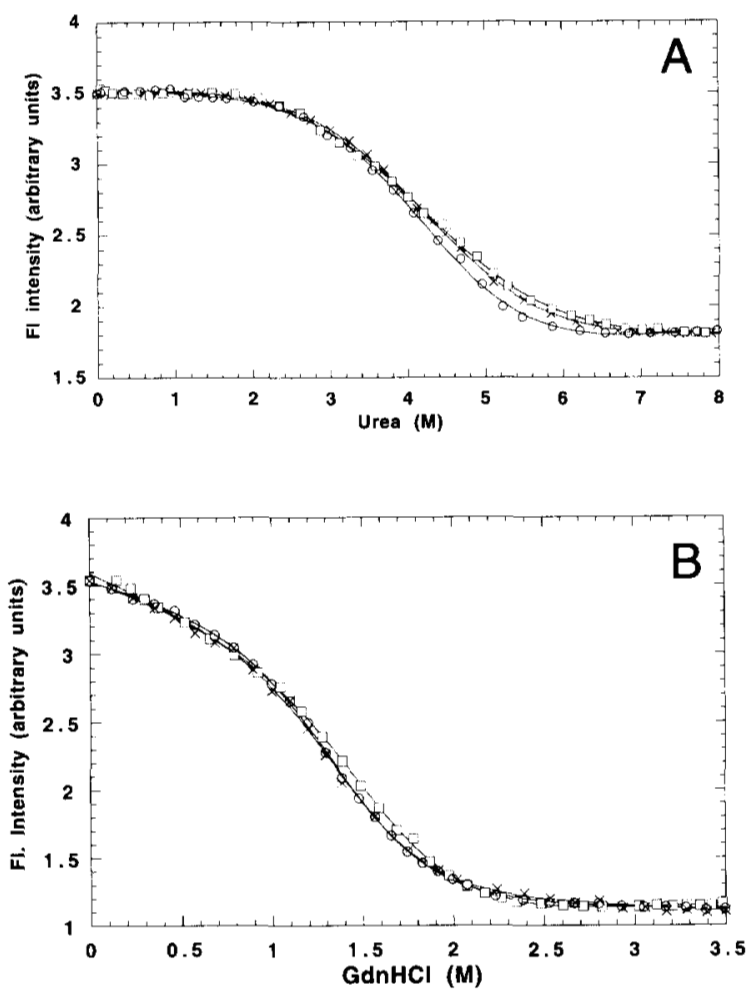

Fig. 2. A: Urea-induced denaturation of CspA variants followed by fluorescence at $25^{\circ} \mathrm{C}$. excitation, $280 \mathrm{~nm}$; excitation slit, $2.5 \mathrm{~mm}$; emission $349 \mathrm{~nm}$; emission slit, $2.5 \mathrm{~mm}$. Protein concentration was $7.0 \mu \mathrm{M}$ in $50 \mathrm{mM}$ potassium phosphate, $100 \mathrm{mM} \mathrm{KCl}, \mathrm{pH} 7.0$ (Buffer $B$ ). $(\times)$ Wildtype CspA; (square) H6-Xa-CspA; (circle) CspA-H6. B: GdnHCl-induced denaturation of $\mathrm{CspA}$ variants followed by fluorescence at $25^{\circ} \mathrm{C} ;(\mathrm{x})$ Wildtype CspA; (square) H6-Xa-CspA; (circle) CspA-H6. Protein concentration was $7.0 \mu \mathrm{M}$ in Buffer B.
These chromophores mask the peptide backbone optical activity typically seen for $\beta$-sheet proteins. The aromatic side chains have been shown to play a major role in the far-UV region of several other proteins including avidin (Green \& Melamed, 1966), soybean and limabean trypsin inhibitor (Woody, 1994), cobratoxin (Dufton \& Hider, 1983), and CspB (Schindler et al., 1995). The same mean residue ellipticity was observed for $\mathrm{CspA}$ at a concentration of $5 \mu \mathrm{M}$ (not shown) as at $50 \mu \mathrm{M}$, implying that there is no structural change in this concentration range as might be caused by protein oligomerization.

To determine the reversibility of thermal denaturation, the three CspA variants were denatured by equilibration at $84^{\circ} \mathrm{C}$ for $30 \mathrm{~min}$. $\mathrm{CD}$ spectra were compared at $10^{\circ} \mathrm{C}$ before and after denaturation. Figure 3 shows the far UV-CD spectra of wild-type CspA before and after thermal denaturation. Identical spectra were observed for the histidine tagged variants. In all three cases, at least $98 \%$ of the $\mathrm{CD}$ signal was recovered after thermal denaturation, demonstrating that the refolding of $\mathrm{CspA}$ is reversible and that the histidine tags do not affect this reversibility. Furthermore, the folding reaction remained reversible when the protein concentration was varied from 17 to $60 \mu \mathrm{M}$, suggesting that aggregation does not play a role in this range.

The thermal stability of the CspA variants was determined by monitoring the change in ellipticity at $222 \mathrm{~nm}$ as a function of temperature in the range from 2 to $90^{\circ} \mathrm{C}$ (Fig. 4). The denaturation midpoint $\left(T_{m}\right)$ was equivalent for all three variants $\left(T_{m}=58.6 \pm\right.$ $1.0^{\circ} \mathrm{C}$ ). This is $4^{\circ}$ lower than observed previously (Chatterjee et al., 1993). A single transition was observed between the folded and unfolded states for the three variants.

One criterion for a two-state folding mechanism is identical denaturation behavior observed by multiple techniques (Eftink, 1995). To this end, we have used circular dichroism spectroscopy to study the equilibrium unfolding of $\mathrm{CspA}$ as a function of urea (Table 3)

Table 2. Midpoint of denaturation, $\Delta G_{H_{2}}$, and $m_{e q}$ values for $\mathrm{GdnHCl}$ denaturation followed by fluorescence at 10 and $25^{\circ} \mathrm{C}$

\begin{tabular}{|c|c|c|c|c|c|c|}
\hline \multirow{2}{*}{$\begin{array}{l}\text { Temperature } \\
\left({ }^{\circ} \mathrm{C}\right)\end{array}$} & \multicolumn{2}{|c|}{$[\mathrm{GdnCl}]_{1 / 2}(\mathbf{M})$} & \multicolumn{2}{|c|}{$\Delta G_{\mathrm{H}_{2} \mathrm{O}}(\mathrm{kcal} / \mathrm{mol})$} & \multicolumn{2}{|c|}{$m_{e q}(\mathrm{kcal} / \mathrm{mol} * \mathrm{M})$} \\
\hline & 10 & 25 & 10 & 25 & 10 & 25 \\
\hline Wild-type CspA & $1.8 \pm 0.2$ & $1.4 \pm 0.1$ & $3.1 \pm 0.2$ & $3.1 \pm 0.1$ & $1.7 \pm 0.2$ & $2.2 \pm c$ \\
\hline H6-Xa-CspA & $1.6 \pm 0.1$ & $1.6 \pm 0.1$ & $3.8 \pm 0.2$ & $3.2 \pm 0.2$ & $2.4 \pm 0.2$ & $2.0 \pm 0$ \\
\hline CspA-H6 & $1.6 \pm 0.1$ & $1.4 \pm 0.1$ & $3.3 \pm 0.1$ & $3.0 \pm 0.1$ & $2.1 \pm 0.1$ & $2.2 \pm c$ \\
\hline
\end{tabular}




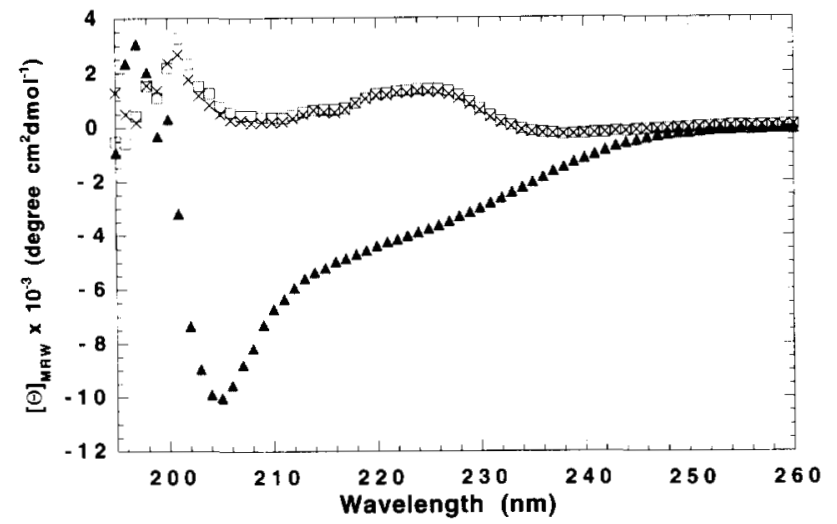

Fig. 3. Far UV-CD spectra of wild-type CspA at different temperatures; (square) $10^{\circ} \mathrm{C}$; (triangle) $84^{\circ} \mathrm{C} ;(\times) 10^{\circ} \mathrm{C}$ after exposure to $84^{\circ} \mathrm{C}$ for $30 \mathrm{~min}$. The path length of the cuvette was $1 \mathrm{~mm}$ and the protein concentration was $50 \mu \mathrm{M}$ in Buffer $\mathrm{B}$.

and $\mathrm{GdnHCl}$ (Table 4) concentration to complement the fluorescence experiments. Because of the weak signal at $222 \mathrm{~nm}$ and manual mixing, the urea and $\mathrm{GdnHCl}$ titrations had a larger experimental error than observed for the titrations followed by fluorescence, which underwent constant stirring (Tables 1 and 2). Despite these technical difficulties, urea and $\mathrm{GdnHCl}$ denaturation followed by $\mathrm{CD}$ were consistent with our data from fluorescence at $25^{\circ} \mathrm{C}$.

\section{Folding followed by stopped flow fluorescence}

The folding kinetics of CspA were followed by monitoring the change in fluorescence of Trp 11. Unfolding and refolding were initiated by a 10 - or 11-fold dilution of the native and unfolded proteins, respectively, to the desired final urea concentration. Under all the conditions observed, the unfolding and refolding were well modeled by monoexponential time courses (Fig. 5). Fitting the kinetic data to a biphasic exponential time course did not result in a better fit, as the rate constant obtained for the second phase

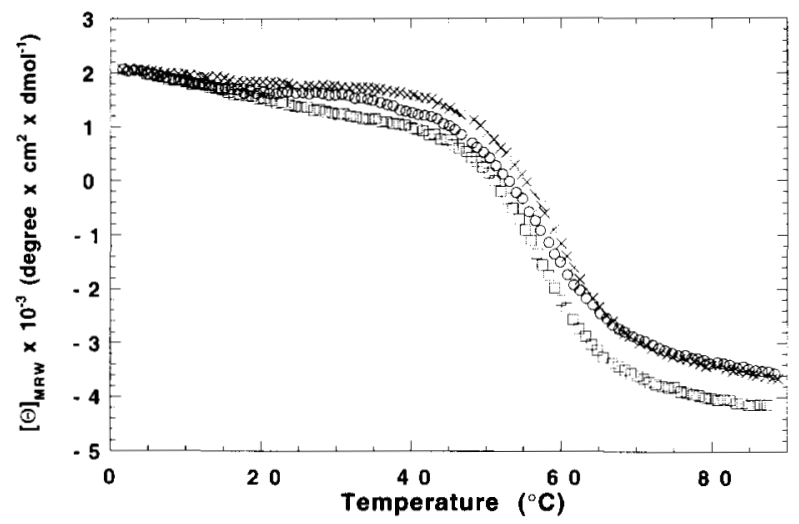

Fig. 4. Thermal stability of $E$. coli CspA variants in buffer B followed by far UV-CD; mean residue weight ellipticity at $222 \mathrm{~nm}$ as a function of temperature. $(\times$ ) Wild-type CspA; (square) H6-Xa-CspA; (circle) CspAH6. Temperature was increased from 2 to $90^{\circ} \mathrm{C}$ at a rate of $0.33^{\circ} \mathrm{C} / \mathrm{min}$. Protein concentration was $50.0 \mu \mathrm{M}$ in buffer containing $50 \mathrm{mM}$ potassium phosphate, $100 \mathrm{mM} \mathrm{KCl}$, pH 7.0.
Table 3. Midpoint of denaturation, $m_{e q}$, and $\Delta G_{\mathrm{H}_{2} \mathrm{O}}$, values for urea denaturation followed by circular dichroism spectroscopy at $25^{\circ} \mathrm{C}$

\begin{tabular}{lccc}
\hline & $\begin{array}{c}{[\text { urea }]_{1 / 2}} \\
(\mathrm{M})\end{array}$ & $\begin{array}{c}\Delta G_{\mathrm{H}_{2} \mathrm{O}} \\
(\mathrm{kcal} / \mathrm{mol})\end{array}$ & $\begin{array}{c}m_{\text {eq }} \\
(\mathrm{kcal} / \mathrm{mol} / \mathrm{M})\end{array}$ \\
\hline Wild-type CspA & $4.0 \pm 1.1$ & $2.6 \pm 0.5$ & $0.6 \pm 0.1$ \\
H6-Xa-CspA & $4.5 \pm 1.1$ & $2.6 \pm 0.5$ & $0.6 \pm 0.1$ \\
CspA-H6 & $4.1 \pm 0.9$ & $3.1 \pm 0.4$ & $0.7 \pm 0.1$ \\
\hline
\end{tabular}

was negative. The dependence of the measured rate constants $(\lambda)$ as a function of urea concentration at $25^{\circ} \mathrm{C}$ for wild-type CspA are shown in Figure 6. Typically, the apparent rate constants $(\lambda)$ follow a V-shaped dependence on denaturant concentration, the apex being the midpoint of denaturation (Tanford, 1970; Matthews \& Hurle, 1987). However, the apparent rate constants for CspA unfolding showed virtually no dependence on urea concentration at concentrations above $4 \mathrm{M}$, causing the chevron plot to resemble a "lazy L" rather than the characteristic "V" shape. The same phenomenon was also observed for the B. subtilis homologue, CspB (Schindler et al., 1995; Schindler \& Schmid, 1996).

All three CspA variants exhibited rapid unfolding and refolding behavior (Table 5). Wild-type and H6-Xa-CspA had comparable rates of refolding and unfolding at 10 and $25^{\circ} \mathrm{C}$. CspA-H6, however, is predicted to re-fold slightly more rapidly $\left(271 \mathrm{~s}^{-1}\right)$ than wild-type CspA $\left(199 \mathrm{~s}^{-1}\right)$ in the absence of denaturant.

The kinetic data are consistent with a two-state folding mechanism. Unfolding and refolding were reversible at 10 and $25^{\circ} \mathrm{C}$ between 0.7 and $7 \mathrm{M}$ urea based on the result that the same rate constants were observed at $4.0 \mathrm{M}$ urea (Fig. 6), which is close to the equilibrium transition midpoint of $4.2 \pm 0.1 \mathrm{M}$ urea. Also, there was no loss in fluorescence amplitude for unfolding or refolding.

\section{Discussion}

Effect of histidine affinity tags on the biophysical properties of CspA

It is often assumed that affinity tags added to proteins are not structured in solution. Because of the frequent use of such tags, we examined their effects on the equilibrium stability and folding properties of CspA. Milla and co-workers have studied the effects of polyhistidine affinity tags on the properties of Arc repressor

Table 4. Midpoint of denaturation, $m_{e q}$, and $\Delta G_{H_{2}} \mathrm{O}$ values for $\mathrm{GdnHCl}$ denaturation followed by circular dichroism spectroscopy at $25^{\circ} \mathrm{C}$

\begin{tabular}{lccc}
\hline \hline & $\begin{array}{c}\Delta G_{\mathrm{H}_{2} \mathrm{O}} \\
(\mathrm{M})\end{array}$ & $\begin{array}{c}m_{e q} \\
(\mathrm{kcal} / \mathrm{mol})\end{array}$ & $(\mathrm{kcal} / \mathrm{mol} / \mathrm{M})$ \\
\hline Wild-type CspA & $1.5 \pm 0.4$ & $2.4 \pm 0.5$ & $1.7 \pm 0.3$ \\
H6-Xa-CspA & $1.3 \pm 0.2$ & $3.7 \pm 0.5$ & $2.9 \pm 0.4$ \\
CspA-H6 & $1.6 \pm 0.3$ & $3.3 \pm 0.4$ & $2.1 \pm 0.3$ \\
\hline
\end{tabular}



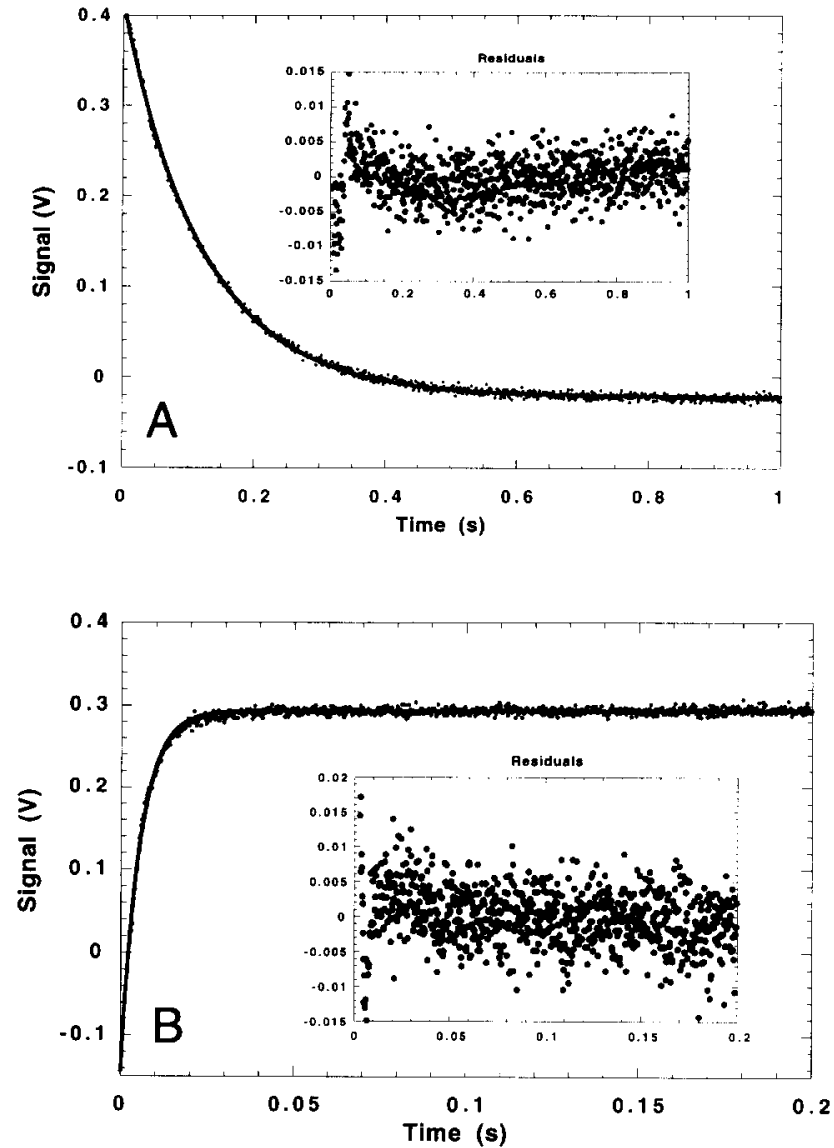

Fig. 5. Kinetic traces for unfolding (A) and refolding (B) of wild-type CspA. Residuals of the signal are shown in the insets. Unfolding was initiated by an 11 -fold dilution of $C \operatorname{spA}$ in buffer $B$ to a final urea concentration of 6.4 M. Refolding was initiated by an 11 -fold dilution of CspA in $7 \mathrm{M}$ urea to a final urea concentration of $0.64 \mathrm{M}$. The final protein concentration for both experiments was $5.1 \mu \mathrm{M}$. The observed amplitude and an apparent rate constant $(\lambda)$ were $0.43 \mathrm{~V}$ and $8.1 \mathrm{~s}^{-1}$ for unfolding and $0.44 \mathrm{~V}$, and $182 \mathrm{~s}^{-1}$ for refolding.

(Milla et al., 1993, 1995). The tags were found to have little effect on the stability or folding of this protein. It is not known, however, whether this result is general. It may be the case that a protein like CspA, which binds single-stranded nucleic acids, may be more

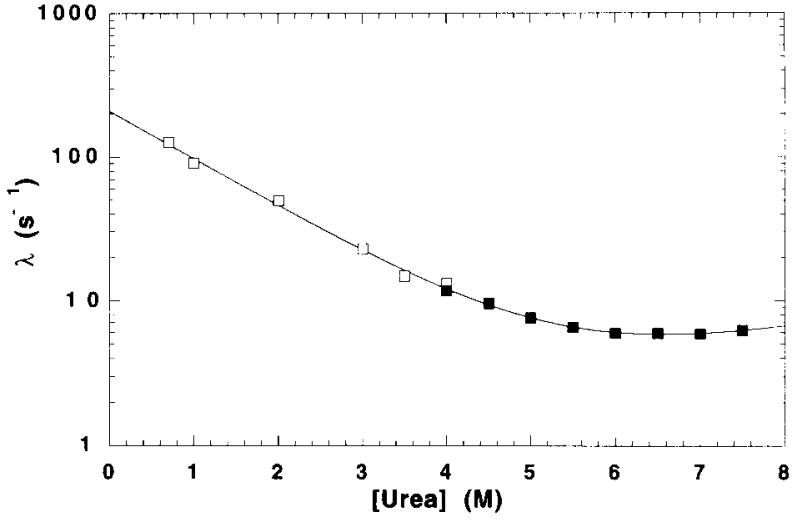

Fig. 6. The dependence of the apparent rate constant $(\lambda)$ on urea concentration for wild type CspA; unfolding (filled symbol); refolding (open symbol). Final CspA concentrations after 10-fold dilution were $5.0 \mu \mathrm{M}$. The kinetics were monitored by the change in fluorescence above $325 \mathrm{~nm}$ in buffer B; excitation was $280 \mathrm{~nm}$. The apparent rate constants are the average of at least 12 kinetic traces. The unfolding and refolding observed rate constants $(\lambda)$ versus urea concentration were fit to the sum of Equations $5 \mathrm{a}$ and $5 \mathrm{~b}$.

sensitive to polyhistidine tags because they resemble the ligand. The affinity tags comprise a significant percentage of the CspA sequence: almost $9 \%$ of the residues of CspA-H6 and $14 \%$ of the residues of $\mathrm{H} 6-\mathrm{Xa}-\mathrm{CspA}$. The tags may alter either the folding behavior or spectroscopic properties of the protein by interacting with the wild-type portion of CspA or by adopting a defined structure.

All three CspA variants are comparable in terms of stability measured by thermal, guanidine hydrochloride, and urea denaturation at $25^{\circ} \mathrm{C}$. Although the stabilities were similar, $\mathrm{CD}$ analysis of the thermal denaturation of the three proteins revealed that the $\mathrm{N}$-terminal tag is not structurally silent, but may contribute a small amount of additional structure, as evidenced by the larger overall change in ellipticity upon unfolding (Fig. 4). One would expect that the addition of 10 residues (six histidines plus four residues comprising the Factor $\mathrm{Xa}$ site) at the amino terminus of a protein would be unstructured. If these 10 residues contained no structure in the native protein, we would expect that the overall change in mean residue ellipticity would decrease slightly. The observed increase may indicate that some of these are in a helical or $\beta$-sheet

Table 5. Kinetics of CspA unfolding and refolding in urea ${ }^{\mathrm{a}}$

\begin{tabular}{|c|c|c|c|c|c|c|c|}
\hline \multirow{2}{*}{$\begin{array}{l}\text { Temperature } \\
\left({ }^{\circ} \mathrm{C}\right)\end{array}$} & \multicolumn{2}{|c|}{ Wild-type CspA } & \multicolumn{2}{|c|}{ H6-Xa-CspA } & \multicolumn{2}{|c|}{$\mathrm{CspA}-\mathrm{H} 6$} & \multirow[b]{2}{*}{ Error } \\
\hline & 10 & 25 & 10 & 25 & 10 & 25 & \\
\hline$k_{u}\left(\mathrm{H}_{2} \mathrm{O}\right)\left(\mathrm{s}^{-1}\right)$ & 3.3 & 4.2 & 3.0 & 4.9 & 2.6 & 3.2 & \pm 7 \\
\hline$k_{f}\left(\mathrm{H}_{2} \mathrm{O}\right)\left(\mathrm{s}^{-1}\right)$ & 188 & 199 & 170 & 224 & 189 & 271 & \pm 14 \\
\hline$m_{u}$ & 0.07 & 0.05 & 0.06 & 0.02 & 0.03 & 0.19 & \pm 0.07 \\
\hline$m_{f}$ & -0.69 & -0.72 & -0.92 & -0.82 & -0.91 & -0.72 & \pm 0.1 \\
\hline$m_{e q}$ & 0.43 & 0.45 & 0.55 & 0.50 & 0.53 & 0.54 & \pm 0.20 \\
\hline$\alpha^{\ddagger}$ & 0.10 & 0.07 & 0.06 & 0.03 & 0.03 & 0.22 & \pm 0.13 \\
\hline
\end{tabular}

${ }^{\text {a }}$ Values were obtained from the kinetic two-state analysis (Equations $5 \mathrm{a}$ and $5 \mathrm{~b}$ ) of the chevron plots. $m_{u}$ and $m_{f}$ values were obtained by extrapolation to $0 \mathrm{M}$ denaturant. $m_{e q}$ and $\alpha^{\ddagger}$ values were obtained from Equations 6 and 7 , respectively. 
conformation. However, this additional structure does not affect the thermal denaturation midpoint of the protein. Kinetic analysis repeatedly demonstrated that $\mathrm{CspA}-\mathrm{H} 6$ refolds slightly faster than the other two variants at $25^{\circ} \mathrm{C}\left(k_{f}=271 \mathrm{~s}^{-1}\right)$. This faster refolding rate was not observed at $10^{\circ} \mathrm{C}$. Although this increased rate may not be significant, it may indicate that the histidines on the C-terminal end act as an intra-molecular catalyst that facilitates the folding process.

The equilibrium and kinetic studies presented here show that the affinity tags affect the biophysical properties of CspA only modestly, even though they comprise a significant portion of the whole protein. Therefore, studies on variants of tagged CspA should be comparable with those on analogous wild-type CspA mutants.

\section{CspA folding is consistent with a two-state mechanism}

All kinetic and equilibrium experiments performed on CspA suggest that it folds by a two-state process where only the native and the denatured states are significantly populated. Our equilibrium denaturation experiments show an unfolding curve with a single transition. The same unfolding transition was obtained by two independent methods, fluorescence and far UV-CD. Although the CD spectrum of CspA is dominated in the far-UV region by the aromatic cluster that includes fluorescent Trp 11, a loss or retention of secondary structure non-concomitantly with the dissolution of the aromatic cluster may be observed as either a non-cooperative transition, or as a much broader transition leading to inconsistent values of [urea $]_{1 / 2}$ and/or $\Delta G_{\mathrm{H}_{2} \mathrm{O}}$. However, both spectroscopic methods gave comparable free energy values $\left(\Delta G_{\mathrm{H}_{2} \mathrm{O}}=3.0 \mathrm{kcal} /\right.$ $\mathrm{mol}$ ) and [urea $]_{1 / 2}$ values $(4.2 \mathrm{M})$. The single unfolding curve was observed independent of the denaturant or denaturation method, i.e., urea, $\mathrm{GdnHCl}$, or heat. Furthermore, the thermal denaturation of $\mathrm{CspA}$ is at least $98 \%$ reversible. Taken together, the equilibrium unfolding data obtained by the two different spectroscopic methods are consistent with a two state model of unfolding, at least at steady state (Eftink, 1995).

It is not uncommon, however, for proteins that exhibit two state unfolding behavior at equilibrium, to have kinetically identifiable intermediates (Jennings \& Wright, 1993; Khorasanizadeh et al., 1996). CspA does not have any detectable kinetic intermediates on the millisecond or greater time scale. Both the unfolding and refolding of CspA monitored by stopped-flow fluorescence are monophasic and well described by monoexponential time dependences. The rate constants for unfolding and refolding are linearly dependent on the concentration of denaturant (Fig. 6). Another important criterion for the two-state argument is close agreement of the $m$-values obtained from equilibrium and kinetic experiments (Equation 6). CspA exhibits an atypical dependence on urea for unfolding. The $m_{u}$ values observed from kinetic experiments are small. Although inconsistency in the $m$-values from kinetic and equilibrium experiments usually suggests the presence of intermediates, in the case of CspA, the small $m_{u}$ values and the error associated with fitting a curve to a small slope complicates comparison of $m_{e q}$ values for kinetic and equilibrium experiments. The equilibrium and kinetic $m$ values ( $\mathrm{kcal} \mathrm{mol}{ }^{-1} \mathrm{M}^{-1}$ ) at $25^{\circ} \mathrm{C}$ are as follows: 0.71 and 0.45 for wild-type CspA; 0.78 and 0.54 for CspA-H6; 0.69 and 0.50 for $\mathrm{H} 6-\mathrm{Xa}-\mathrm{CspA}$. However, the urea denaturation midpoint ([urea $]_{1 / 2}$ ) of wild-type CspA is in excellent agreement with kinetic experiments. Finally, the amplitudes of the kinetic traces account for the entire fluorescence change expected (Fig. 5): no burst phase was observed.
The above points are strong evidence for a two-state mechanism. However, inherent limitations in kinetic measurements detect only intermediates with stabilities greater than $10 \%$ of the native state (Jackson \& Fersht, 1991). Therefore, highly unstable intermediates may still be present on the folding pathway. Also, transient intermediates that may be present within the dead time of the experiments $(2 \mathrm{~ms})$ would not be detected.

\section{CspA folds rapidly without intermediates}

CspA is a $\beta$-sheet protein that folds rapidly with no detectable intermediates. This observation is interesting from two standpoints. First, because of the long chain separation between residues in $\beta$-sheets, proteins containing this type of secondary structure have been expected to fold more slowly than helical proteins. Our observations of CspA do not fulfill this expectation. CspA folds very rapidly - its extrapolated refolding rate in the absence of denaturant at $25^{\circ} \mathrm{C}$ is $199 \mathrm{~s}^{-1}$. This rate is comparable to only two other $\beta$-sheet proteins studied thus far: B. subtilis CspB (Schindler et al., 1995) and ubiquitin (Khorasanizadeh et al., 1996). Although it may turn out that the upper limit for fast folding is intrinsically higher for all-helical proteins than for all-sheet proteins (Burton et al., 1996), millisecond folding is clearly possible for $\beta$-sheet proteins. Second, CspA folds fast in the absence of intermediates. CspB folds even more rapidly without detectable intermediates (Schindler et al., 1995; Schindler \& Schmid, 1996). This lack of intermediates along the folding pathways of the cold shock proteins is not entirely surprising, because they have only one domain and no disulfide bonds. It does, however, contradict the hypothesis that intermediates are necessary to direct a protein to the final folded conformation (Kuwajima et al., 1987; Kim \& Baldwin, 1990 ) and that intermediates are required for rapid $\beta$-sheet folding (Rudolph et al., 1990; Ropson \& Frieden, 1992; Varley et al., 1993; Liu et al., 1994).

\section{CspA has a compact transition state}

Although CspA appears to fold without intermediates, it is still possible to envision its folding pathway by studying the transition state of folding. The solvent accessibility of the transition state $\left(\alpha^{\ddagger}\right)$ may be inferred from the dependence of the folding and refolding rate constants on urea concentration as reflected in Equation 7 (Tanford, 1970; Chen et al., 1989). The low value of $\alpha^{\ddagger}$ calculated for CspA suggests that its ensemble of transition state structures resembles the native state much more closely than the denatured state. For wild-type CspA, $\alpha^{\ddagger}=0.07$, implying that the transition state is $93 \%$ native-like. Although Burton and coworkers have noted the lack of a relationship between the folding rate constant and the compactness of the transition state (Burton et al., 1996), we believe that in this case, the unusually compact transition state is correlated with the observed rapid folding of CspA and its homologue CspB. For both of these proteins, there is essentially no dependence on urea concentration above $4.0 \mathrm{M}$ $\left[m_{u}=0.05 \mathrm{kcal} \mathrm{mol}^{-1} \mathrm{M}^{-1}\right.$ for CspA and $0.03 \mathrm{kcal} \mathrm{mol}^{-1} \mathrm{M}^{-1}$ for CspB (Schindler \& Schmid, 1996)] indicating that the native state and the activated states of folding interact similarly with the denaturant.

An unusual structural feature of $\operatorname{CspA}$ is the large, solvent accessible cluster of aromatic residues on $\beta$-strands one, two, and three. Six aromatic residues contribute to this cluster, which to- 
gether with other nonpolar atoms, create a continuous nonpolar patch of $790 \AA^{2}$, comprising $19 \%$ of the total surface area of CspA. This is unusual, particularly for such a small protein, because nonpolar patches greater than $350 \AA^{2}$ are highly uncommon (Lijnzaad et al., 1996). Although this aromatic patch is undoubtedly involved in nucleic acid binding (Newkirk et al., 1994), it may also direct folding. Tisi and Evans have observed that smaller solvent exposed hydrophobic clusters are often seen in proteins with $\beta$-barrel architectures and postulate that these clusters may assist in the correct alignment of $\beta$-strands (Tisi \& Evans, 1995). We speculate that coalescence of the surface aromatic residues occurs in the folding transition of CspA. The conserved aromatic cluster in the cold shock proteins may therefore serve a dual purpose in ligand binding as well as rapid folding. It is worth noting that the $\mathrm{SH} 3$ domain of spectrin is another small $\boldsymbol{\beta}$-barrel protein that has a cluster of aromatic amino acids on its surface (mostly tyrosines) that are involved in polyproline peptide binding. This protein also re-folds rapidly and via a compact transition state (Viguera et al., 1994).

To test whether the aromatic cluster aids in nucleating the folding of CspA, we examined the refolding of wild-type CspA from 7 to $0.64 \mathrm{M}$ urea in the presence of a 15-mer deoxyoligonucleotide ( $K_{\mathrm{D}}=6 \mu \mathrm{M}$; Hillier et al., in press). We hypothesized that folding would be accelerated because the ligand would stabilize a transition state in which the aromatic cluster is formed. We observed a faster rate of folding in the presence of the $15-\operatorname{mer}\left(\lambda=230 \mathrm{~s}^{-1}\right)$ compared to an apparent rate constant of $131 \mathrm{~s}^{-1}$ under the same conditions in the absence of the oligonucleotide. Additionally, of 14 aromatic cluster mutants investigated thus far, all are destabilizing (Hillier et al., in press), implying that the residues responsible for the protein's function are also necessary for stability. Although these preliminary results support our hypothesis, additional experiments, including more extensive ligand binding studies together with an analysis of the effects of aromatic cluster mutations on the transition state, are necessary to determine whether the aromatic cluster is indeed critical to the rapid folding of CspA.

\section{Materials and methods}

\section{Materials}

Guanidine hydrochloride $(\mathrm{GdnHCl}) 8.0 \mathrm{M}$ sequenal grade was obtained from Pierce. Urea analytical grade was obtained from ICN. The components of all other buffers and purification reagents were of analytical grade and purchased from Fisher. Ni-NTA agarose used to purify the histidine-tagged CspA was purchased from Qiagen (Germany). Factor Xa protease used to cleave the affinity tag was purchased from New England Biolabs. Protein concentration was determined using a UV-visible Hewlett-Packard 8452A diode array spectrophotometer. The extinction coefficient was calculated to be $8,437 \mathrm{M}^{-1}$ (Pace et al., 1995).

\section{Cloning of CspA variants}

The gene coding for CspA with six histidines at the carboxy terminus (CspA-H6) was created synthetically and subsequently ligated into the vector pAD100 (Davidson \& Sauer, 1994). Expression of CspA-H6 protein is controlled by a hybrid trp/lac repressorbased trc promoter. The plasmid (pLG200) was introduced into the E. coli strain $\mathrm{X} 90$ by electroporation. The gene sequence was verified using the dideoxy chain termination method (Sanger et al., 1977). The gene for CspA with N-terminal histidines and the Factor Xa cleavage site (H6-Xa-CspA) was created by PCR using the above-described C-terminal six histidine construct as a template. The nonannealing 5' end of the primer for the beginning of the new gene was designed to code for the amino acids $M(H)_{6}$ IEGR. The primer for the $3^{\prime}$ end was designed to omit the $C$-terminal $\mathrm{H}_{6}$ tag. The PCR product was cloned into the polylinker region of the plasmid pAED4 (Doerring, 1992), contains a T7 polymerasebased promoter. This plasmid $(\mathrm{pBH} 1)$ was transformed into the E. coli strain BL21(DE3). The gene for the C-terminal histidinetagged CspA was also subsequently cloned into the vector pAED4 using a similar PCR method.

\section{Protein purification}

Cells with the plasmid containing the $\operatorname{csp} A$ gene of interest were grown to an $\mathrm{OD}_{600}$ of 0.6 then induced with $1 \mathrm{mM}$ IPTG for $3 \mathrm{~h}$. From an original culture volume of $6 \mathrm{~L}$, the cell pellets were resuspended in $40 \mathrm{~mL}$ of Buffer A (6 M GdnHCl, $0.1 \mathrm{M} \mathrm{NaH}_{2} \mathrm{PO}_{4}$, $10 \mathrm{mM}$ Tris; $\mathrm{pH} \mathrm{8.0)}$ and stirred at room temperature for $1 \mathrm{~h}$. The lysate was cleared by centrifugation at $15,000 \times g$, and the supernatant loaded on a 1 -mL Ni-NTA column equilibrated with Buffer A. The column was washed with $100 \mathrm{~mL}$ of Buffer $\mathrm{A}$. The protein was eluted with $15 \mathrm{~mL}$ of Buffer $F(6 \mathrm{M} \mathrm{GdnHCl}, 0.2 \mathrm{M}$ acetic acid) and dialyzed against $4 \mathrm{~L}$ of Buffer $\mathrm{B}(50 \mathrm{mM}$ potassium phosphate, $100 \mathrm{mM} \mathrm{KCl}, \mathrm{pH} 7.0$ ) with one exchange. Wild-type CspA was obtained by cleaving the histidine tag with Factor Xa protease at a 1:100 $(\mathrm{w} / \mathrm{w})$ ratio for $15 \mathrm{~h}$ at room temperature and then separated via a G-75 (Pharmacia) size exclusion column. The molecular mass of CspA-H6 was determined using a MicroMass Quattro II electrospray mass spectrometer. The mass obtained (8094 amu) was consistent with complete processing of the $\mathrm{N}$-terminal methionine.

\section{$\mathrm{GdnHCl}$ and urea-induced denaturation followed by fluorescence}

Fluorescence measurements were performed using a Spex Fluorolog 2 or Spex Fluoromax-2 by exciting the single tryptophan of CspA (Trp 11) at $280 \mathrm{~nm}$ and monitoring its emission at $349 \mathrm{~nm}$ (wavelength-corrected spectra). A 2.5-mm slit width was used for the excitation and emission beams. A 1-mm sample containing $7.0 \mu \mathrm{M}$ protein in Buffer B was placed in a $1-\mathrm{mL}$ fluorescence quartz cuvette. The sample was continually mixed throughout the experiment using a magnetic stir bar (Labcraft). A thermostatted cuvette holder was used to maintain a temperature of 10 or $25^{\circ} \mathrm{C}$. $\mathrm{GdnHCl}$ and urea titrations were performed by removing an aliquot of protein solution and replacing the same volume with a solution containing identical protein concentration in $6 \mathrm{M} \mathrm{GdnHCl}$ or $9 \mathrm{M}$ urea. The folding equilibrium was reached rapidly as determined with stopped-flow fluorescence experiments. The urea and $\mathrm{GdnHCl}$ stock solutions were prepared fresh daily and the concentrations were calculated by index of refraction (Nozaki, 1972) using a Bausch \& Lomb refractometer. To obtain values for $\Delta G_{\mathrm{H}_{2} \mathrm{O}}$ and $m_{e q}$ (slope of the transitional region), a two-state transition was assumed and the relative changes in fluorescence intensity with increasing denaturant were calculated using the Pace method (Pace, 1986) by a six-parameter fit to the following equation using Kaleidagraph for the Macintosh (variable parameters are italicized in the text): 


$$
I=\frac{\left(m_{f} \cdot[D]+y_{f}\right)+\left(m_{u} \cdot[D]+y_{u}\right) \cdot \exp \left(\frac{-\Delta G_{\mathrm{H}_{2} \mathrm{O}}+m_{e q} \cdot[D]}{R T}\right)}{1+\exp \left(\frac{-\Delta G_{\mathrm{H}_{2} \mathrm{O}}+m_{e q} \cdot[D]}{R T}\right)}
$$

where $I$ is the signal intensity, $m_{f}$ and $m_{u}$ are the slopes of the folded and unfolded baselines, $y_{f}$ and $y_{u}$ are the intercepts of the folded and unfolded baselines, $[D]$ is the denaturant concentration, $K$ is the equilibrium constant, $f_{u}$ is the unfolded fraction of molecules, $\Delta G_{u}$ is the free energy of unfolding, $\Delta G_{\mathrm{H}_{2} \mathrm{O}}$ is the extrapolated free energy of unfolding in water (in the absence of denaturant) and $m_{e q}$, the " $m$ value," is the dependence of $\Delta G_{u}$ on urea. The concentration at the midpoint of denaturation, [denaturant $]_{1 / 2}$, also known as the $C_{m}$, is given by

$$
[\text { denaturant }]_{1 / 2}=\frac{\Delta G_{\mathrm{H}_{2} \mathrm{O}}}{m_{e q}}
$$

\section{Circular dichroism studies}

Far UV-circular dichroism (CD) denaturation experiments were performed on an Aviv Associates (Lakewood, NJ) model 60DS instrument. Urea and $\mathrm{GdnHCl}$ denaturation assays were performed at $25^{\circ} \mathrm{C}$. A $1-\mathrm{mm}$ path length cuvette was used to minimize absorbance by the denaturant. A similar titration method as employed for fluorescence was used for denaturation unfolding followed by far UV-CD except the CD cuvette was manually inverted several times to ensure mixing. The protein concentrations were between 35 and $50 \mu \mathrm{M}$ in buffer $\mathrm{B}$, and kept constant for a given titration. The change in ellipticity at $222 \mathrm{~nm}$ was monitored as a function of denaturant concentration. Each titration point was the average of 60 data points recorded every second using a $1.5-\mathrm{nm}$ bandwidth.

The thermal denaturation of $\mathrm{CspA}$ variants were determined by measuring the change in ellipticity at $222 \mathrm{~nm}$ with increasing temperature. The temperature was increased at a constant rate of $0.33^{\circ} \mathrm{C} / \mathrm{min}$ using a programmable water bath. The spectrometer was equipped with a temperature probe that was calibrated to an external probe $\left( \pm 0.1{ }^{\circ} \mathrm{C}\right)$. Temperature and ellipticity at $222 \mathrm{~nm}$ were recorded every $3 \mathrm{~min}$ from 2 to $90^{\circ} \mathrm{C}$, and each of the data points were averaged over $35 \mathrm{~s}$. Thermal denaturation curves were fit using a nonlinear least-squares method using the Pace method (Pace et al., 1989).

Far UV-CD spectra were recorded from 190 to $260 \mathrm{~nm}$. Data were collected every $1 \mathrm{~nm}$, with a $5 \mathrm{~s}$ averaging time and $1.5-\mathrm{nm}$ bandwidth. Five wavelength scans were averaged to obtain each spectrum. Equilibrium unfolding experiments using fluorescence and far UV-CD were performed at least twice; averaged values are reported.

\section{Stopped flow fluorescence}

Folding and unfolding reactions were followed using a Biologic SFM-2 stopped-flow apparatus employing Biokine 3.0 software (Claix, France) or a DX.17MV sequential mixing stopped-flow from Applied Photophysics (Leatherhead, UK). The dead time of both stopped-flow instruments was determined to be approximately 2 ms. Using the Biologic SFM-2, unfolding of CspA was initiated by a rapid 10 -fold dilution with different urea concentra- tions in buffer B to a final protein concentration between 4 and $6 \mu \mathrm{M}$. Final urea concentrations for unfolding after dilution were $4.0,4.5,5.0,5.5,6.0$, and $7.0 \mathrm{M}$. The fluorescence emission increase associated with CspA refolding was monitored using $50 \mu \mathrm{M}$ CspA in $7 \mathrm{M}$ urea stock solution. Re-folding was initiated upon a 10-fold dilution giving a final protein concentration of $5 \mu \mathrm{M}$ and final urea concentrations of $0.7,2,3,3.5$, and $4 \mathrm{M}$. Each kinetic measurement consisted of a $100 \mu \mathrm{L}$ sample containing $10 \mu \mathrm{L}$ of the protein stock and $90 \mu \mathrm{L}$ of the urea stock solution. The fastest mixing time of $6 \mu \mathrm{L} / \mathrm{ms}$ was used in all kinetic experiments. Experiments using Applied Photophysics stopped-flow were performed in a similar manner except an 11-fold dilution was used, resulting in final urea concentrations between 4-6.4 $\mathrm{M}$ and 0.64 $4 \mathrm{M}$ for unfolding and refolding, respectively. The rates of the unfolding and refolding reactions were determined by fitting the average of the data to a monoexponential curve using Kaleidagraph software. The fluorescence change was monitored using a $325 \mathrm{~nm}$ filter following excitation at $280 \mathrm{~nm}$ using a $2-\mathrm{mm}$ slit width. The path length and the volume of the cuvettes were $2 \times$ $2 \times 10 \mathrm{~mm}$ and $90 \mu \mathrm{L}$, respectively. All samples were filtered through a $0.45-\mu \mathrm{m}$ filter and degassed for $30 \mathrm{~min}$ to reduce noise. The apparent rate constants $(\lambda)$ are the average of at least 12 kinetic traces. Kinetic measurements were consistent for both stopped-flow instruments.

Refolding of CspA (50 $\mu \mathrm{M})$ in the presence of single-stranded DNA $(100 \mu \mathrm{M})$ was initiated upon an 11-fold dilution from $7 \mathrm{M}$ to $0.64 \mathrm{M}$ urea. Applied Photophysics stopped-flow parameters were the same as above. The sequence of the single-stranded oligonucleotide was 5'-CTTGAGGTTAATCCA-3'. Fluorescence binding studies (K. Reid, unpubl. results) revealed that this particular 15-mer binds CspA with higher affinity than its complement.

\section{Analysis of the folding kinetics}

The folding and unfolding reaction for CspA was modeled as a two-state reaction:

$$
N \underset{k_{f}}{\stackrel{k_{u}}{\rightleftharpoons}} D
$$

The apparent rate constant, $\lambda$, is equal to the sum of the microscopic rate constants for refolding, $k_{f}$, and unfolding $k_{u}$ :

$$
\lambda=k_{u}+k_{f}
$$

The natural logarithms of $k_{f}$ and $k_{u}$ will vary linearly with the urea concentration. For a two-state folding mechanism, the relation for unfolding is

$$
\ln k_{u}=\ln k_{u}\left(\mathrm{H}_{2} \mathrm{O}\right)+\left(m_{u} \cdot[\text { urea }]\right)
$$

and for folding

$$
\ln k_{f}=\ln k_{f}\left(\mathrm{H}_{2} \mathrm{O}\right)+\left(m_{f} \cdot[\text { urea }]\right)
$$

The $m_{u}$ and $m_{f}$ values describe the denaturant dependence of the folding or unfolding reactions and are thought to be proportional to the change in buried surface area that occurs between the folded or unfolded state and the transition state. The $m_{u}$ and $m_{f}$ values were obtained by the sum of Equations $5 \mathrm{a}$ and $5 \mathrm{~b}$, excluding the points 
in the transition region (Chen et al., 1992; Viguera et al., 1995). If the folding and unfolding of a protein follow a two-state mechanism, then the $m$ values obtained from equilibrium and kinetic data should be equal within experimental error:

$$
m_{e q}=\left(m_{u}-m_{f}\right) \cdot R T
$$

The solvent accessibility of the transition state is determined by $\alpha^{\ddagger}$ :

$$
\alpha^{\ddagger}=\frac{m_{u}}{m_{u}-m_{f}}=\frac{R T m_{u}}{m_{e q}}
$$

which describes the fractional change in solvent upon moving from the native state to the transition state relative to the total change upon unfolding (Tanford, 1970; Chen et al., 1989).

\section{Acknowledgments}

This work was funded by grants from the NIH (GM52885-R29), and the University of California Cancer Research Coordinating Committee, and by startup funds from UCSC. Hector Rodriguez is supported by a GAANN fellowship. We would like to thank Bob Sauer and Chuck Wilson for DNA synthesis reagents and synthesizer use, Alan Davidson for plasmid pAD 100 , Tony Fink and Roberto Bogomolni for use of their fluorimeters, Jon Goldberg and the Baldwin lab for use of and assistance with their stopped flow fluorimeter, Frank Eisenhaber for solvent accessibility calculations on CspA. and Franz Schmid, Daniel Palleros, Glenn Millhauser, and Susan Marqusee for helpful comments on the manuscript.

\section{References}

Alexander P, Orban J, Bryan P. 1992. Kinetic analysis of folding and unfolding the 56 amino acid IgG-binding domain of streptococcal protein G. Biochemistry 31:7243-7248

Blanco F, Rivas G, Serrano L. 1994. A short linear peptide that folds into a native stable beta-hairpin in aqueous solution. Nat Struct Biol 1:584-590.

Burton RE, Huang GS, Daugherty MA, Fullbright PW, Oas TG. 1996. Microsecond protein folding through a compact transition state. J Mol Biol 263:31 1322

Chatterjee S. Jiang W, Emerson SD, Inouye M. 1993. The backbone structure of the major cold shock protein CS7.4 of Escherichia coli in solution includes extensive $\beta$-sheet structure. $J$ Biochem 114:663-669.

Chen B, Baase WA. Schellman JA. 1989. Low-temperature unfolding of a mutant of phage T4 lysozyme. 2. Kinetic investigations. Biochemistry 28:691-699.

Chen BL, Baase WA, Nicholson H, Schellman JA. 1992. Folding kinetics of T4 lysozyme and nine mutants at 12 degrees C. Biochemistry 31:1464-1476

Clark PL, Liu ZP, Zhang J, Gierasch LM. 1996. Intrinsic tryptophans of CRABPI as probes of structure and folding. Protein Sci 5:1108-1117

Davidson AR, Sauer RT. 1994. Folded proteins occur frequently in libraries of random amino acid sequences. Proc Natl Acad Sci USA 91:2146-2150.

Dobson C, Evans P, Radford S. 1994. Understanding how proteins fold: The lysozyme story so far. Trends Biochem Sci 19:31-37.

Doerring D. 1992. Functional and structural studies of a small $[$-actin binding domain. PhD, Massachusetts Institute of Technology.

Dufton MJ, Hider RC. 1983. Conformational properties of the neurotoxins and cytotoxins isolated from Elapid snake venoms, $C R C$ Crit Rev Biochem $14: 113-171$

Eftink MR. 1995. Use of multiple spectroscopic methods to monitor equilibrium unfolding of proteins. Methods Enzymol 259:487-512

Goldstein J, Pollitt NS, Inouye M. 1990. Major cold shock protein of Esche richia coli. Proc Natl Acad Sci USA 87:283-287.

Green NM, Melamed MD. 1966. Optical rotatory dispersion, circular dichroism and far-ultraviolet spectra of avidin and streptavidin. Biochemical J 100:614621.

Hamada D, Segawa S, Goto Y. 1996. Non-native alpha-helical intermediate in the refolding of beta-lactoglobulin, a predominantly beta-sheet protein. Nat Struct Biol 3:868-73.

Hillier BJ, Rodriguez HM, Gregoret LM. Coupling of protein stability and function in E. coli CspA. Fold \& Des. In press.
Hooke S, Radford S, Dobson C. 1994. The refolding of human Jysozyme: A comparison with the structurally homologous hen lysozyme. Biochemistr $33: 5867-5876$.

Jackson SE, Fersht AR. 1991. Folding of chymotrypsin inhibitor 2. 1. Evidence for a two-state transition. Biochemistry 30:10428-435.

Jennings PA, Wright PE. 1993. Formation of a molten globule intermediate carly in the kinetic folding pathway of apomyoglobin. Science 262:892-896.

Jiang W, Hou Y, Inouye M. 1997. CspA, the major cold shock protein of Escherichia coli, is an RNA chaperone. J Biol Chem 272:196-202

Khorasanizadeh S, Peters ID, Roder H. 1996. Evidence for a three-state model of protein folding from kinetic analysis of ubiquitin variants with altered core residues. Nat Struct Biol 3:193-205.

Kiefhaber T. 1995. Kinetic traps in lysozyme folding. Proc Natl Acad Sci USA 92:9029-9033.

Kim PS, Baldwin RL. 1990. Intermediates in the folding reactions of small proteins. Annu Rev Biochem 59:631-660.

Kragelund B, Hojrup P, Jensen M. Scherling C, Juul E, Knudsen J, Poulsen F. 1996. Fast and one-step folding of closely and distantly related homologous proteins of a four helix bundle family. $J \mathrm{Mol}$ Biol 256:187-200.

Kraulis PJ. 1991. MOLSCRIPT - A program to produce both detailed and schematic plots of protein structures. J Appl Crystallogr 24:946-950.

Kuwajima K, Yamaya H, Miwa S, Sugai S, Nagamura T. 1987. Rapid formation of secondary structure framework in protein folding studied by stopped flow circular dichroism. FEBS Lett 221:115-118.

Lijnzaad P, Berendsen HJC, Argos P. 1996. Hydrophobic patches on the surfaces of protein structures. Proteins 25:389-397.

Liu ZP, Rizo J, Gierasch LM. 1994. Equilibrium folding studies of cellular retinoic acid binding protein, a predominantly beta-sheet protcin. Biochemistry 33:134-142.

Makhatadze G, Maraheil M. 1994. Effect of pH and phosphate ions on selfassociation properties of the major cold shock protein from Bacillus subrilis. Protein Sci 3:2144-2147.

Matouschek A, Otzen DE, Itzhaki LS, Jackson SE, Fersht AR. 1995. Movement of the position of the transition state in protein folding. Biochemistry 34:13656-1.3662.

Matthews CR, Hurie MR. 1987. Mutant sequences as probes of protein lolding mechanisms. Bioessays 6:254-257.

Milla ME, Brown BM, Sauer RT. 1993. P22 Arc repressor: Enhanced expression of unstable mutants by addition of polar C-terminal sequences. Protein Sci 2:2198-2205

Milla ME, Brown BM, Waldburger CD, Sauer RT. 1995. P22 Arc repressor: Transition state properties inferred from mutational effects on the rates of protein unfolding and refolding. Biochemistry 343:13914-13919.

Nesloney C, Kelly J. 1996. Progress towards understanding $\beta$-sheet structure Bioorgan Med Chem 4:739-766.

Newkirk K, Feng W, Jiang W, Tejero R, Emerson SD, Inouye M, Montelione GT. 1994. Solution NMR structure of the major cold shock protein (CspA) from Escherichia coll: Identification of a binding epitope for DNA. Proc Nat! Acad Sri USA 9!5114-5118.

Nozaki Y. 1972. The preparation of guanidine hydrochloride. Methods Enzymol $26: 43-50$

Pace CN. 1986. Determination and analysis of urea and guanidine hydrochloride denaturation curves. Methods Enzymol 131:266-280.

Pace CN, Shirley BA. Thomson JA. 1989. Measuring the conformational stability of a protein. In: Creighton T, ed. Protein structure: A practical approach. Oxford, UK: IRL Press, pp .311-330.

Pace CN, Vajdos F, Fee L, Grimsley G, Gray T. 1995. How to measure and predict the molar absorption cocfficient of a protein. Protein Sci 4:241 1-2423.

Ramirez-Alvarado M. Blanco FJ, Serrano L. 1996. De novo design and structural analysis of a model beta-hairpin peptide system. Nat Struct Biol 3:604-612.

Ropson IJ, Frieden C. 1992. Dynamic NMR spectral anaiysis and protein folding: Identification of a highly populated folding intermediate of rat intestinal fatty acid-binding protein by 19F NMR. Proc Natl Acad Sci USA 89:72227226

Ropson IJ, Gordon JI. Frieden C. 1990). Folding of a predominantly hetastructure protein: Rat intestinal fatty acid binding protcin. Biochemistr 29:959l-9599.

Rudolph R, Siebendritt R, Nesslauer G, Sharma AK, Jaenicke R. 1990. Folding of an all-beta protein: Independent domain folding in gamma II-crystallin from calf eye lens. Proc Natl Acad Sci USA 87:4625-4629.

Sanger F, Nicklen S, Coulson AR. 1977. DNA sequencing with chain-terminating inhibitors. Proc Natl Acad Sci USA 74:5463-5467.

Schindelin H, Jiang W, Inouye M, Heinemann U. 1994. Crystal structure of CspA, the major cold shock protein of Escherichia coli. Proc Natl Acad Sci USA 91:5119-5123.

Schindelin H, Marahcil MA, Heinemann U. 1993. Universal nucleic acidbinding domain revealed by crystal structure of the $B$. subtilis major coldshock protein. Nature 364:164-168. 
Schindler T, Herrler M, Maraheil MA, Schmid FX. 1995. Extremely rapid protein folding in the absence of intermediates. Nature Struct Biol 2:663-673.

Schindler T, Schmid FX. 1996. Thermodynamic properties of an extremely rapid protein folding reaction. Biochemistry 35:16833-1642.

Searle M, Williams D, Packman L. 1995. A short linear peptide derived from the $\mathrm{N}$-terminal sequence of ubiquitin folds into a water-stable non-native $\beta$-hairpin. Nat Struct Biol 2:999-1006.

Tanford C. 1970. Protein denaturation. C. Theoretical models for the mechanism of denaturation. Adv Protein Chem 24:1-95.

Tisi LC, Evans PA. 1995. Conserved structural features on protein surfaces: Small exterior hydrophobic clusters. J Mol Biol 249:251-258.
Varley P, Gronenborn AM, Christensen H, Wingfield PT, Pain RH, Clore GM. 1993. Kinetics of folding of the all $\beta$ sheet protein interleukin- $1 \beta$. Science 260:1110-1113.

Viguera AR, Blanco FJ, Serrano L. 1995. The order of secondary structure elements does not determine the structure of a protein but does affect its folding kinetics. J Mol Biol 247:670-681.

Viguera AR, Martinez JC, Filimonov VV, Mateo PL, Serrano L. 1994. Thermodynamic and kinetic analysis of the $\mathrm{SH} 3$ domain of spectrin shows a twostate folding transition. Biochemistry 33:2142-2150.

Woody RW. 1994. Contributions of tryptophan side chains to the far-ultraviolet circular dichroism of proteins. Eur Biophys $J$ 23:253-262. 